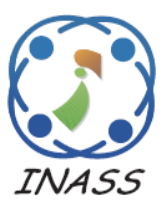

\title{
Intelligent Adaptation of Axisymmetric Solid Parts in Machining Process Planning by Case Based Reasoning
}

\author{
Alexis Cordovés García ${ }^{1 *}$, Ricardo Sors Lancho ${ }^{2}$, Arlys M. Lastre Aleaga ${ }^{1}$, Alexis Cordovés Rodríguez ${ }^{3}$, \\ and Leonardo L. Lorente Leyva ${ }^{4}$ \\ ${ }^{1}$ Equinoccial Technological University, Santo Domingo, Ecuador \\ ${ }^{2}$ Cane Harvesters KTP' Factory, Holguín, Cuba \\ ${ }^{3}$ CE-CAD/CAM, Holguín University, Holguín, Cuba \\ ${ }^{4}$ North Technical University, Ibarra, Ecuador \\ * Corresponding author’s Email: alexis.cordoves@ute.edu.ec
}

\begin{abstract}
The goal of this paper is the adaptation of plans of manufacturing processes of Axisymmetric Solid Parts under the approach of Case Based Reasoning (CBR). To this end, a data model for feature-based representation has been developed, which contains the geometric, dimensional and technological information required for the preparation of the one-piece process plan. As a result, an algorithm to generate new parts process plans from a similar plan retrieved from the case base is presented, which is based on the specific information of the Primitive Form Features (PFF) and considers the possible interaction between neighboring PFFs. The computer support of the developed algorithm was implemented in AutoLISP as programming language of the CAD system used and the interface was created in OpenDCL.
\end{abstract}

Keywords: CAD/CAPP system, Cutting schemes, Form features, Geometric information, CBR.

\section{Introduction}

The process of generating the solution to an engineering problem is often based on the use of previous experiences. In some industries these experiences "are stored" in technological documents. According to this principle, Case-Based Reasoning (CBR) is the process of solving new problems based on solutions given to previous problems [1].

CBR techniques have been successful in different application domains. At the Industrial level it has been applied in the modeling of the workflow [2-3]. Chann P., Jie J., and Yuan W. [4] developed a hybrid CBR and Artificial Neural Network (RNA) system to determine the unit cost of manufacturing mobile phones.

The industrial processing of mechanical parts involves a series of human and material resources (machine tools, cutting tools, accessories, lubricants, among others) that must be rationalized to reduce the total costs of production. To this end, the advanced experience of the experts is used, which is regularly stored in the form of technological documents with the description of the operations and the order in which they are to be executed, to transform the blank into finished parts. It is about achieving the best possible solution for the manufacture of the parts according to the existing conditions of production with the associated minimum manufacturing costs.

For the generation of the cylindrical and prismatic pieces cutting process, several authors have focused on the use of rules-based Primitive Form Features (PFF), which contain the information required for their manufacture, and then use Artificial Intelligence techniques (IA), among them the CBR, in order to detect the level of similarity between two EFPs belonging to different pieces (phase of retrieval in the CBR). Basically, the geometric coincidence of the part to be fabricated with the existing ones in the base of cases is verified, without considering 
technological attributes as the precision and type of material [5-9].

Hsin-chi Chang, Wen F. Lu and Xiaoqing Frank Liu [10], worked on the planning of the process of cutting rotational symmetrical parts, and introduced significant improvements on previous systems when considering the precision, material and the cutting process history. However, in determining the overall similarity between two cases, they proposed an expression of similarity, which in regard to the material of the part, only consider the heat treatment and the hardness achieved. In addition to these factors, Sors R., Cordovés A., Avila R., García R., Yarza J.A., Esteves E. and Lastres A.M. [11] considered the type of material, type of stock and its dimensions.

Once the most similar part to the new one is identified from the database, that part is recovered with its corresponding manufacturing process plan. The required modifications (adding and / or subtracting operations) are made to the recovered process plan until it is adapted to the requirements of the new part. The present research is focused specifically on the adaptation stage within the CBR.

This research will focus specifically on the adaptation stage within the CBR.

\section{Materials and Methods}

In general, a case is the description of a problem and its solution. In the present investigation, it consists of the description of a Axisymmetric Solid Part and its cutting technology. The part is made up of EFPs which are elementary sections of it with design and manufacturing data.

Parametric representation of parts using PFF. A PFF is an object that can store information about its functionality, location, material, dimensions and tolerances, surface roughness and its relationship with other elements [12]. Each PFF is assigned a unique code called primitive form feature code. A sample of the PFFs included in a library is presented in Fig.1.

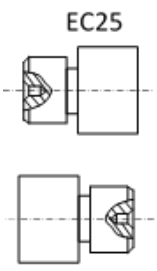

EC05
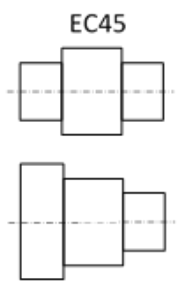

EC44
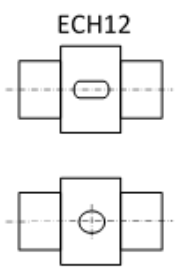

EC48
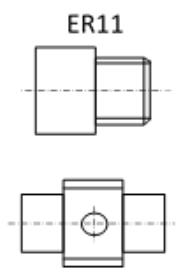

EE11
Figure.1 Primitives form features with their code
Peripheral Cutting Index (ICP-2D). The arrangement of each step with respect to the larger diameter step will influence the order in which the technological steps are carried out. Based on this observation, the (PCI-2D) is developed considering the diameters and their relative arrangement. Thus the PCI-2D index: 3-2-1-3-2-4-3 corresponds to the part of Fig. 2 and indicates that the larger diameter with value (1) has an intermediate position. To each form feature corresponds a digit, the first value (3), followed by (2), which indicates that the step (b) will be elaborated earlier than (a) from the larger diameter step (c) with digit equals to 1 . In the steps located to the right of the largest step (c) it was preceded in the same way, which shows that the non-contiguous steps (d) and (g) have the same digit (3), that is, they may have the same priority when they are machined if two variants were used for this case (c-e-g-d-f) or (c-e-d-g-f).

Representation of the process plans. In the present investigation, the cut history is defined from the succession of technological steps to convert the previous state of the PFFs (stock) into their final state (finished part), ensuring the technical and accuracy requirements (See Fig. 3).

Each technological step, the type of accuracy (IT) and the surface roughness value (Rz) that it can reach is assigned. This information is associated with the PFF in the database.

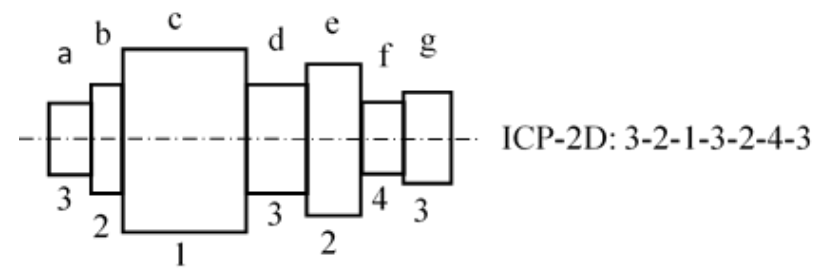

Figure. 2 Value of Peripheral Cutting Index

\section{$\mathrm{ECH} 12$}

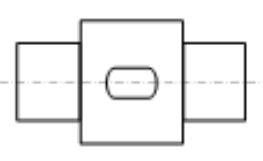

Technological Steps for PFF:

ECH12-1: Rough turning

ECH12-2: Finish turning

ECH12-3: Keyway milling

ECH12-4: Cylindrical grinding

Figure.3 Succession of technological steps to develop the PFF-ECH12 


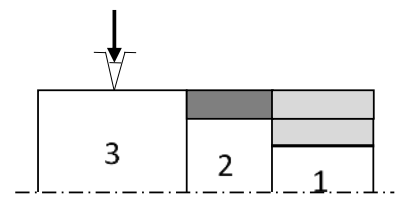

(a)

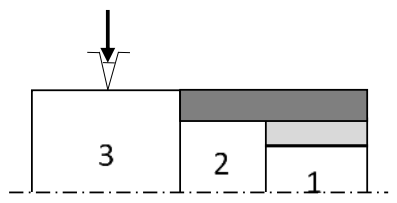

(b)
Figure.4 Cutting schemes for a combination of EFPs:

(a) step 1 and (b) step2

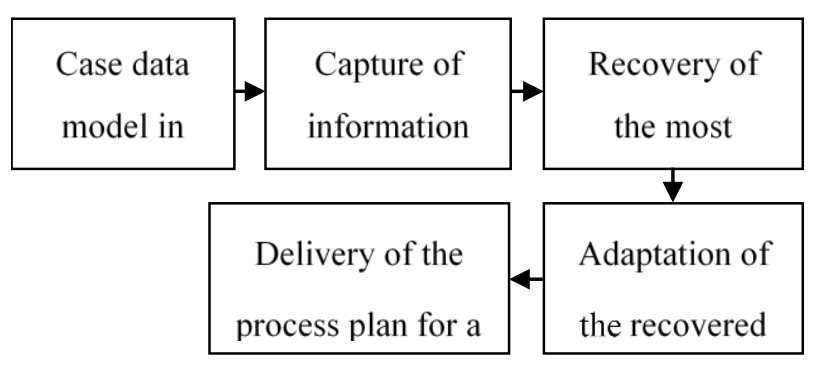

Figure. 5 General structure of the system for the adaptation of manufacturing process plan for

Axisymmetrical parts by CBR.

For the definition of the cutting scheme to be followed, an algorithm that considers the technological base (s) used, the diametrical relationships between the neighboring PFFs, the surface condition to be achieved and the cutting times involved is proposed. In Fig. 4 two cutting schemes for the same combination of PFF are shown. Each step of the part constitutes an independent PFF. Step 3 is used as a technological base in both cases, step 1 can be machined separately Fig. 4 (a) or in conjunction with the neighboring PFF, step 2, Fig. 4 (b). A system for obtaining the cutting plan must select the best cutting scheme under technicaleconomic considerations. In general, the steps are machined from the largest diameter to the smallest diameter, finishing work and delicate surfaces, such as threads, are processed at the end of the process.

\subsection{System structure for the adaptation of process plans of axisymmetrical parts, using CBR}

The Fig. 5 shows the structure of the system for obtaining manufacturing process plan of axisymmetrical parts.

a)Case data model in the case base. The efficiency and quality of the results delivered by the CAPP system will depend on the data structure and the information management algorithm used. A case is represented as the sum of the part information and its cutting process plan. In turn, the part will contain information of itself and the stock which it is manufactured.
Case $=$ Part + Manufacturing process plan

Part $=$ Finished part + Stock

Finished part $=\{\mathrm{ICP}-2 \mathrm{D},(\mathrm{PFFi},(\mathrm{Di}, \mathrm{j} ; \mathrm{Li}, \mathrm{j} ; \mathrm{Rzi}, \mathrm{j}$;

ITi,j)) $\forall \forall \mathrm{j} \in \mathrm{I} \mathrm{i} ; \mathrm{i}=1, \ldots$, it;

Stock $=\{$ Mht, HB, Tm, Fb, Dm $\}$

Manufacturing process plan $=\{$ MTi $($ TecBasj, $\mathrm{i}$

(Tsk,j (Vk, fk, tk, Pask), Ctk, Acck, Refk)) $\} \forall \mathrm{i} \in$ I $\mathrm{m} ; \mathrm{m}=1, \ldots, \mathrm{mt} ; \forall \mathrm{j} \in \mathrm{I} \mathrm{n} ; \mathrm{n}=1, \ldots, \mathrm{nt} . ; \forall \mathrm{k} \in \mathrm{I} \mathrm{p}$; $\mathrm{p}=1, \ldots, \mathrm{pt}$;

Where: PCI-2D: Peripheral Cutting Index; D:diameter; L: Length of elaboration; Rz: Surface roughness; IT: Precision; Mht: Heat treatment; HB: Hardness; Tm: Material; Fb: Stock; Dm: Longwide relationship of stock; MT: Machine tool; TecBas: Technological base; Ts: Technological step; $\mathrm{V}$ :Cutting speed ( $\mathrm{m} / \mathrm{min})$; f:feed $(\mathrm{mm} / \mathrm{rev})$; t:Cutting depth; Pas: Cutting passes; Ct: cutting tool; Acc: Accessory; Ref: refrigerant.

b) Capture of information for a new case. In Fig. 6a) the dimensional description of a part is shown, in Fig. 6b) the profile from which the 3D part was drawn in the CAD system, Fig. 6c). It also indicates its GFI and the Secondary Index. The capture of the dimensional information of the PFF is performed automatically when traversing the vertices of the profile of the part drawn in AutoCAD Fig.6b). For this, an application was developed in AutoLISP language, obtaining directly the diameters, lengths and angles that define the contour of the part, keyways, spline, holes and threads, contained in each PFF. Surface roughness and precision values are entered by the user. The information is stored in the extended database of drawing entities.

c) Recovery of the most similar case from the case base. The present research focuses on the adaptation of manufacturing process plans for this reason it will not cover the part recovery stage.

d) Adaptation of the recovered case. As the new part does not completely coincide with the geometrical and dimensional requirements of the recovered part, adaptations must be made to the recovered plan, which consists of adding or subtracting technological steps, or both, to satisfy the specific requirements of the new part. The following is the algorithm used to adapt the cutting plan, which presupposes the use of the same type of stock for the part recovered and the new part: It has been implemented using a computer tool developed in AutoLisp for AutoCAD work environment. 


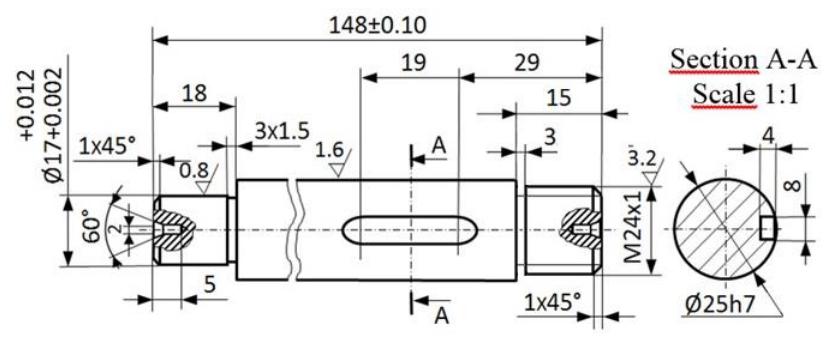

(a)

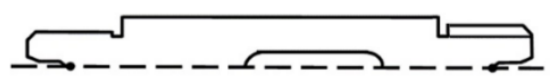

(b)

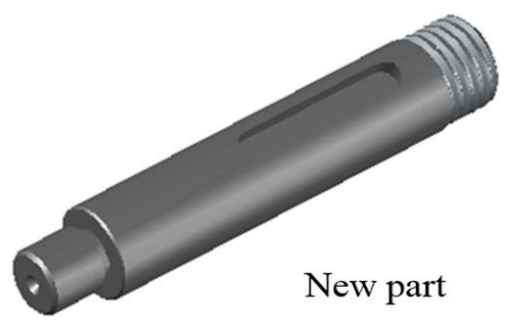

(c)

Figure.6 New part information:

(a) Dimensional descriptions of the part, (b) 2D profile corresponding to the part, and (c) 3D drawing of the part, GFI: EC25_ECH12_ER11

\subsection{Algorithm for the automated generation of the plan of cut of a new part from the recovered plan}

1.Capture the geometric and dimensional information of the new part, modeled in a CAD system.

The dimensional description of the new piece is made by two ways: the capture of the geometric and dimensional information directly from the 2D profile of the new piece and by the user specification of the accuracy indexes of the surfaces (Accuracy class and surface roughness).

2. Generate the ICP-2D of the new part using a CAD system.

Ensure the orientation of the new part with the largest number of steps to the right of the step of greater diameter (this arrangement of steps facilitates its elaboration)

3. Carry out the adaptation process of the recovered plan, attending to the new piece requirements, according to the following cases:

a) Quantity PFF new part $=$ Quantity PFF retrieved part Process: [subtraction/addition]

Retrieved part New part
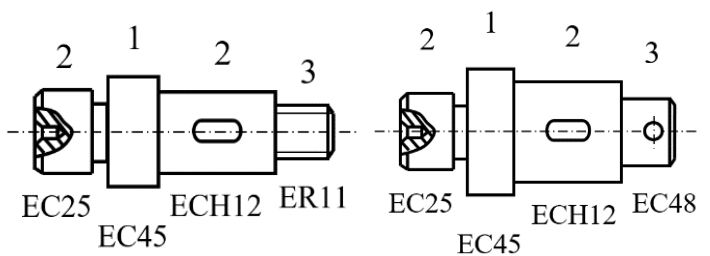

IFG retrieved part: EC25_EC45_ECH12_ER11

IFG new part: EC25_EC45_ECH12_EC48

The PFFs of both pieces are traversed in the order defined by their respective IFG, with a subtraction/addition operation: The technological steps corresponding to the PFFs that do not correspond to their counterpart of the new part are "subtracted" from the recovered part, in the example the PFF ER11 is subtracted. Then the technological steps of the EFPs of the new part replacing those taken from the recovered part, in this case the cylindrical step with through holeEC48, are "added". Only the technological steps required according to the accuracy class and the surface roughness value assigned to the surfaces of the added PFFs will be incorporated.

The order of incorporation of the technological steps for the elaboration of each PFF is made with attention to the placements and to the chosen cutting scheme in the recovered process plan. The system may suggest a different cutting scheme depending on the minimum cut times involved and must be approved by the user when integrating different technological criteria.

b) Quantity PFF new part $>$ Quantity PFF retrieved part Process: [subtraction] Retrieved part
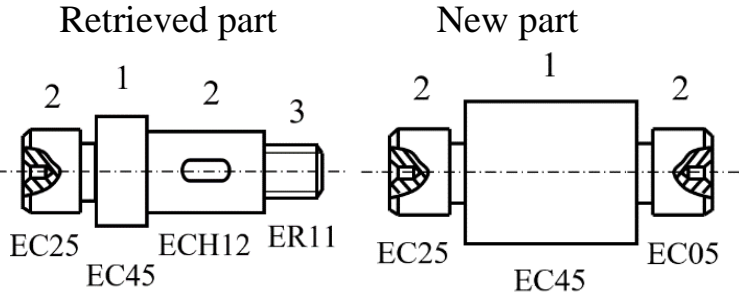

IFG retrieved part: EC25_EC45_ECH12_ER11

IFG new part: EC25_EC45_ECO5

The ICP-2D code of both parts is traversed by comparing each digit from the location of the step with the largest diameter (value 1). In the case where it occupies an intermediate position, the route is initially carried out in the sense that there are fewer digits (corresponds to the smaller number of steps) and then in the other sense. This will allow the identification of the location of the PFFs of the recovered part that do not have their 
counterpart in the new part (PFFs of the recovered part that are in excess) and will not be considered for the preparation of the process plan of the new part.

From the process plan of the recovered part and in correspondence with the ICP-2D code values, the technological steps of the PFFs that are common to both parts are maintained, "subtraction / addition" operations are performed for the different PFFs Which have the same location in the ICP-2D code, as was done in case $3 a)$, the steps of the excess PFFs that were identified in the recovered part are not considered. In the example, the technological steps of the PFFs (EC25, EC45) are maintained and the technological steps of the PFF ECH12 of the part recovered are replaced by those of the PFF EC05 of the new part. The technological steps of PFF ER11 are not considered.

c) Quantity PFF new part < Quantity PFF retrieved part Process: [addition]

Retrieved part

New part

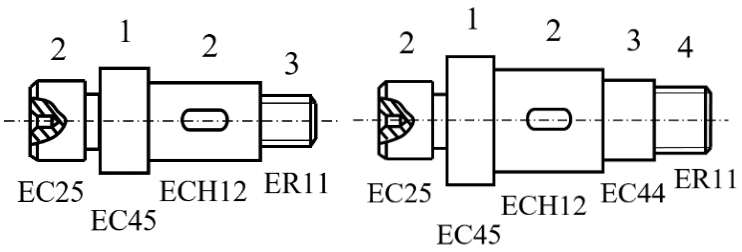

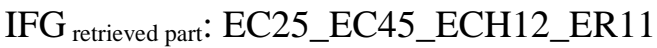

IFG new part: EC25_EC45_ECH12_EC44_ER11

As in case $3 b$ ), the ICP-2D code of both parts is traversed to identify the location of the additional PFFs in the new part. From the process plan of the recovered part and in correspondence with the ICP-2D code values, the technological steps of the PFFs that are common to both parts are maintained, "subtraction / addition" operations are performed for the different PFFs Which have the same location in the ICP-2D code, as was done in case 3a), and the technological steps of the new PFFs are "added" to the plan according to their location in the ICP-2D code. In the example, the technological steps of the PFFs (EC25, EC45, ECH12) are maintained, the technological steps of the PFF ER 11 of the part recovered by those of the PFF EC44 of the new part are "replaced", and the technological steps of the PFF ER11 of the new part are "added".

If there are common technological steps in neighboring EFPs that are analyzed, the cutting scheme that involves them with minimum processing time is determined.

4. Delivery of the cutting plan for the new part, in the information model assumed by the company according to available technology.

d) Delivery of the process plan for the new part. Application of the adaptation stage to a study case. In this section we proceed to apply the algorithm developed to obtain the process plan of the new part shown in Fig 6b). To do this, we will use four parts of the total contained in the case base (See Fig. 7).

By applying the axisymmetry solid recovery procedure described in [11], the most similar part to the new part, out of the four that were evaluated, was CASE01, with a similarity value of 0.92 (See Table $1)$.

Table 2 shows, on the left, the cut-off plan of the recovered case (CASE01) and on the right the modified one for the new part. In the example, the codes of the technological steps followed in each process plan and the action of subtraction and addition are indicated. The new cutting plan is stored in the case database associated with the new part which can then be retrieved for new cases. From it, the definitive plan to manufacture the part is prepared according to the custom format assumed by the company, with the required dimensional and technological details. (TecBas, V, f, t, Pas, Ct, Acc and Ref).
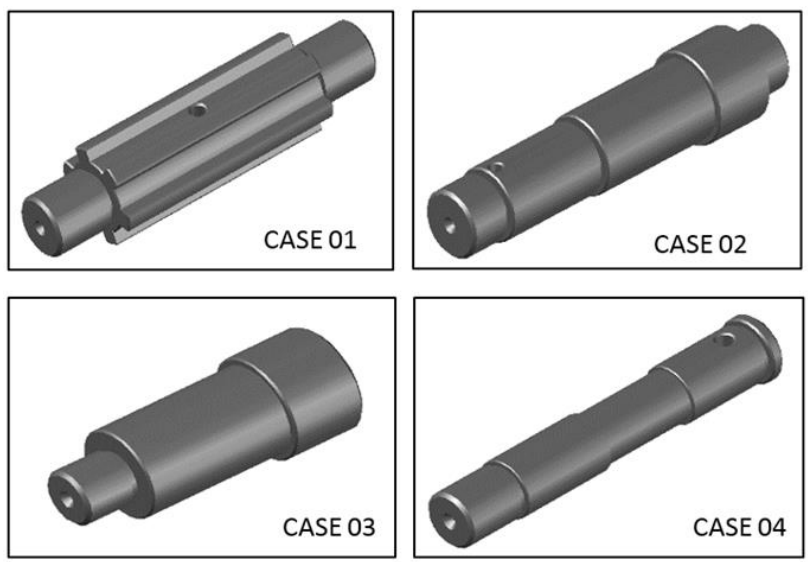

Figure.7 Parts contained in a case base 
Table 1. Global similarity values of the new part with the prototype parts

\begin{tabular}{|c|c|}
\hline Comparison & Similarity Global \\
\hline New part y CASE01 & 0,92 \\
\hline New part y CASE02 & 0,50 \\
\hline New part y CASE03 & 0,15 \\
\hline New part y CASE04 & 0,39 \\
\hline
\end{tabular}

Table 2. Obtaining the cutting plan of the new part from the plan retrieved from CASE 01

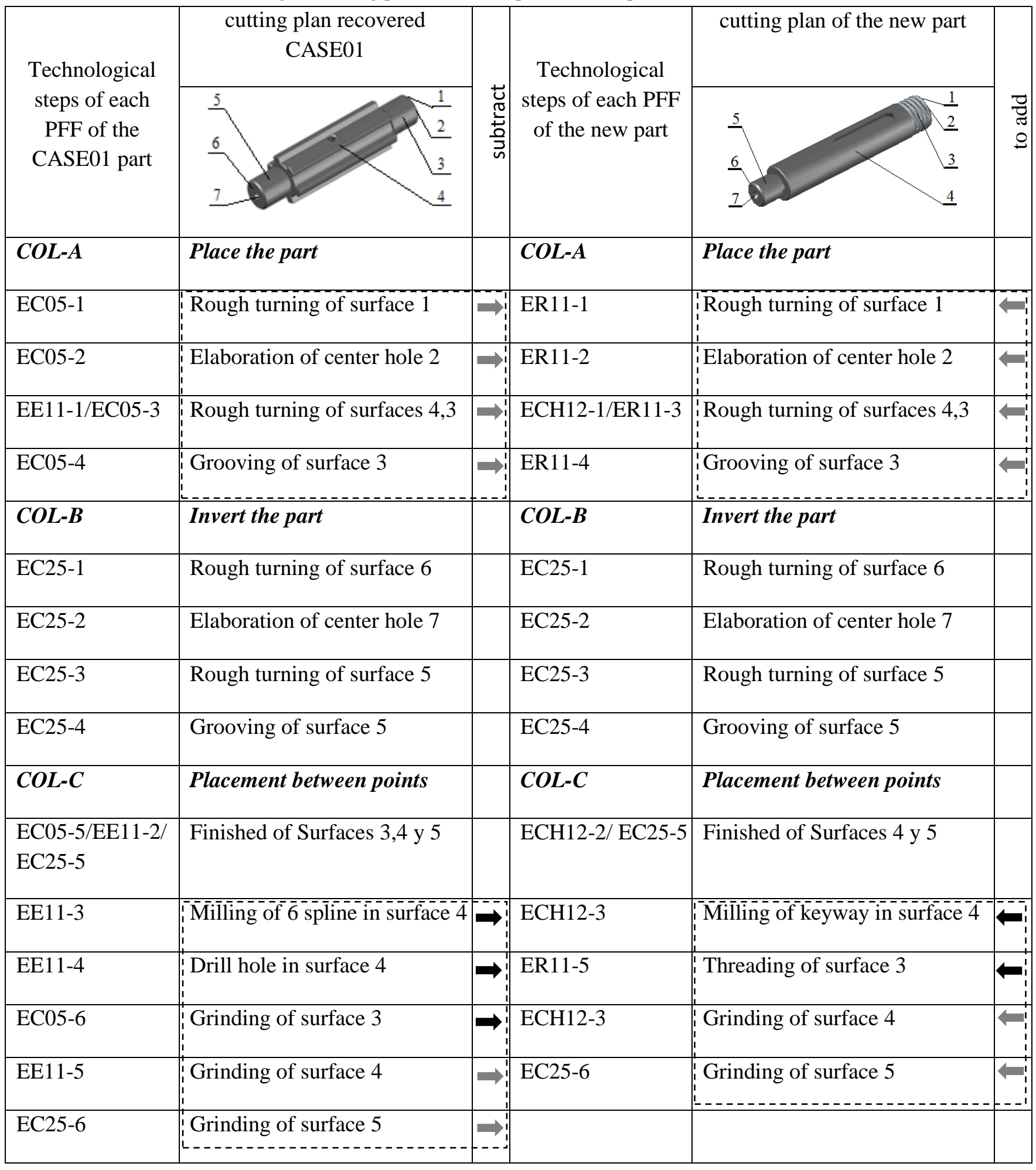




\subsection{Advantages of the application of the algorithm developed}

Among the main merits of the developed proposal are the following:

1. The CBR method allows to obtain new process plans taking advantage of previous work experience, which guarantees its better adaptation to the conditions of the technological equipment installed in the company.

2. The Capturing the design information of the new parts to be manufactured are made in the same working environment of the CAD system in which they were created; this, together with the data structure presented, allows quick access to the specific information of the pieces stored in the extended database of the graphical entities and their respective cutting plans.

3. The delivery of the generated cut plan can adapt to the output formats of the technological information assumed by the user company.

4. The foundations of the present investigation can be applied to the generation of cutting plans for other types of parts and cutting processes.

\section{Results and discussion}

High level of coincidence was observed between the process plans of the recovered part and the one developed for the new part. This was possible thanks to a $92 \%$ similarity between the two parts, supported by the great geometric coincidence (equal quantity and distribution of the steps) and their technological similarities.

Although the IFG of the recovered part and the new part coincided in a single PFF (EC25) and that subtraction / addition operations were performed in the remaining two PFFs, the most significant changes in the process plan for the new part are reduced to those indicated with the intense color arrows, which were:

a) Carry out the subtraction of the technological steps in intermediate step 4, of the recovered part, CASE01, consisting of milling of 6 spline, and drilling of the hole, and in the step of the right end 3 the corresponding to the grinding.

b) Incorporate in the plan of the new part, the technological steps of milling of keyway in the intermediate step 4 , and the threading of the step 3.

The variations in the geometric indexes of both parts corroborate these changes:

Subtraction operation:GFI CASE01EC25_EE11_EC05
Adition operation: GFI New partEC25_ECH12_ER11

The results obtained reaffirm the potential of the integration of the CBR method with efficient forms of representation and storage of information such as the PFF in the manufacturing systems.

As a result, the process plan with the sequential technological steps for the new part from a similar plan retrieved from the base case, was obtained.

\section{Conclusion}

An algorithm to obtain the manufacturing process plan of a new part from the adaptation of the recovered plan of a similar part stored in a case base was presented. The algorithm is based on Case-Based Reasoning (CBR) and on the form features theory.

The data model that has been implemented allows to store the geometric, dimensional and technological information of each PFF with the consecutive technological steps required for its complete manufacture.

The CAD application in which the algorithm was implemented was developed for the AutoCAD working environment using the AutoLISP programming language with an OpenDCL interface, allows an efficient database management and evaluates the most appropriate cutting scheme to manufacture each PFF according to its interaction with neighboring PFFs based on technical-economic criteria. Process plans generated will be exported to an Excell sheet according to the custom format of the Company.

The future development of the present investigation is directed to the extension of the developed algorithm, for the treatment of axisymmetric parts with cavities and solid part in other machining processes.

\section{References}

[1] I. Watson, "Case-Based Reasoning is a methodology not a technology", KnowledgeBased Systems. pp. 303-308, 1999.

[2] T. Madhusudan, J.L. Zhao, and B. Marshall, "A case-based reasoning framework for workflow model management", Data \& Knowledge Engineering, Vol. 50, No. 7, pp. 87-115, 2004.

[3] K. Choy and W. Lee, "Task allocation using casebased reasoning for distributed manufacturing systems", Logistics Information Management, Vol. 13. No. 3, pp. 167-176, 2000.

[4] P. Chann, J. Jie, and W. Yuan, "Forecasting of manufacturing cost in mobile phone products by case-based reasoning and artificial neural 
network models", Journal of Intelligent Manufacturing, pp. 517-531, 2012.

[5] Y. Hao, F. Wen, and C. Alan, "PROCASE: a case-based process planning system for machining of rotational parts", Journal of Intelligent Manufacturing, pp. 411-430, 1994.

[6] G. Mohammad, A. Haghighi, and L. Wang, "Generic machining process sequencing through a revised enriched machining feature concept", Journal of Manufacturing Systems, Vol. 37, No. 8, pp. 564-575, 2015.

[7] H. Muñoz-Avila, "Integrating Twofold Case Retrieval and Complete Decision Replay in CAPLAN/CBC", Tesis Doctoral. Universität Kaiserslautern, mayo, 1998.

[8] S. Singh and S. Deb, "An intelligent methodology for optimizing machining operation sequence by ant system algorithm", International Journal of Industrial and Systems Engineering, pp. 451-471, 2014.

[9] C. Tsatsoulis and R. Kashyap, "Case-Based Reasoning and Learning in Manufacturing with the TOLTEC Planner", IEEE Transactions on systems, man, and cybernetics, Vol. 23, No. 4, pp. 1010-1023, 1993.

[10] C. Hsin-chi, F. Wen, and F. L. Xiaoqing, "Intelligent Case Retrieval and Modification for Machining Process Planning of Axisymmetric Parts", In: Proc. of the Artificial Intelligence and Manufacturing Workshop. pp. 55-62, 1998.

[11] R. Sors, A. Cordovés, R. Avila, R. García, J.A. Yarza, E. Esteves, and A.M. Lastres, "Intelligent Retrieval of Axisymmetric Solid Parts in Machining Process Planning by Case Based Reasoning", In: Proc. of the 2nd International Conference on Modelling, Identification and Control (MIC 2015), pp. 200-204, 2015.

[12] R.L. Avila, "CAPP based on form features", In: Proc. of the 13th ISPE/IEE International Conference on CAD/CAM Robotics \& Factories of the Future'97, pp. 283-2, 1997. 